\title{
Assessment of Macular Perfusion in Early Diabetic Retinopathy Patient Using Optical Coherence Tomography Angiography
}

\author{
Amr Farahat Ali Farahat ${ }^{1, *}$ MB Bch, Ahmed Mohamed Youssef ${ }^{2}$ MD and Ahmed Nabil El Sayed Hafez ${ }^{2}$
} MD

\author{
* Corresponding Author: \\ Amr Farahat Ali \\ amrfarahat70@gmail.com
}

Received for publication April 10, 2020; Accepted July 15, 2020; Published online July 15, 2020.

\section{Copyright 2020 The Authors} published by Al-Azhar University, Faculty of Medicine, Cairo, Egypt. All rights reserved. This an open-access article distributed under the legal terms, where it is permissible to download and share the work provided it is properly cited. The work cannot be changed in any way or used commercially.

doi: $10.21608 /$ aimj.2020.27644.1195

1 Sheikh Zayed hospital Ministry of health - Sheikh Zayed city, Giza, Egypt.

${ }^{2}$ Ophthalmology Department, Faculty of Medicine, Al-Azhar University, Cairo, Egypt.

\begin{abstract}
Background: The chronic hyperglycemia of diabetes leads to long-term damage, and deterioration of different organs, especially the eyes, kidneys, nerves, and blood vessels. The riskiest complication includes diabetic retinopathy and maculopathy.

Aim of work: Assessment of macular perfusion in early subclinical diabetic retinopathy patients by using noninvasive technology Optical Coherence Tomography Angiography (OCTA), to prevent vision loss.

Patient and methods: A prospective case series observational study includes 44 eyes for 33 candidates, 30 eyes for diabetic Patients and 14 eyes for normal non-diabetic, Patients' Age group is $30-60$ years old. Results: Regarding both groups diabetic and non-diabetic, patients with normal fundus picture, $46.2 \%$ had very mild to mild affection of macular perfusion (SVP-VD $50 \pm 3.5 \%$, DVP-VD $56 \pm 3.0 \%$ ) with FAZ perimeter range (1.3-1.5) mm, 53.8\% did not have macular perfusion affection and none of the patients had moderate affection of macular perfusion. While patients whom fundus picture showing mild-NPDR, $11.1 \%$ had very mild to mild affection of macular perfusion and $88.9 \%$ had moderate affection of macular perfusion (SVP -VD $45 \pm 4.5 \%$, DVP-VD $50 \pm 4.0 \%$ ) with FAZ perimeter range $(1.7-2.8) \mathrm{mm}$, a significant difference between groups (P-value <.0001).

Conclusion: Macular perfusion is markedly affected by diabetes duration despite of good clinical fundus picture. OCTA is very beneficial in investigating diabetic maculopathy and ischemia in early stages.
\end{abstract}

Keywords: Macular Perfusion, Diabetic Retinopathy, Optical Coherence Tomography Angiography.

Disclosure: The authors have no financial interest to declare in relation to the content of this article. The Article Processing Charge was paid for by the authors.

Authorship: All authors have a substantial contribution to the article.

\section{INTRODUCTION}

About 347 million people worldwide suffering diabetes mellitus (DM), and these numbers predicted to be increased to 430 million patients by $2030^{1}$.

The Riskiest factor in diabetic retinopathy (DR) is Macular non-perfusion which is a critical feature that can lead to significant visual impairment. Microvascular dysfunction in DR causes hypoperfusion, retinal hypoxia and at the macula it threatens the tissue responsible for central visual acuity, diabetic patients with poor macular perfusion are often asymptomatic until the later stages when vision loss can be acute and severe. Additionally, advanced macular non-perfusion can limit the benefits of treatment. Therefore, identification and quantification of these microvascular changes in early stages can prevent deterioration of vision ${ }^{2,3,4}$.

Ophthalmologists have used different methods to observe the vasculature in the macular area including fundus photography, and fundus fluorescein angiography (FFA). But these methods are limited due to its invasive nature. Moreover, the monitoring of early and subtle changes in the macular capillary

system in clinical settings has proven to be challenging. Thus, an effective, high resolution, and non-invasive method for monitoring macular perfusion would provide a precise and easy way to understand and observe early perfusion changes ${ }^{5,6}$

Optical coherence tomography angiography (OCTA), a modality that utilizes motion contrast to generate perfusion maps. OCTA able to identify vascular changes in DR, imaged neovascularization, and quantified areas of macular ischemia. Their quantification of macular ischemia includes measuring the diameter of the foveal avascular zone, the total area of vessel non-perfusion, microaneurysms, venous beading, and neovascularization ${ }^{7,8}$.

Moreover, OCTA novel imaging technique obtains high-resolution volumetric blood flow information and generates angiographic images in a matter of seconds. OCT angiograms are resampled with OCT B-scans from the same area, simultaneously allowing the assessment of structure and blood flow ${ }^{\mathbf{9 , 1 0}}$. 


\section{PATIENT AND METHODS}

Patients: this is a prospective case series observational study includes 44 eyes for 33 candidates, in which 30 eyes for diabetic Patients and 14 eyes for normal non-diabetic, as a control group, Patients' Age is $30-60$ years old.

All the patients were subjected to medical history taking, Visual Acuity assessment, Intraocular pressure measurement, and slit-lamp biomicroscope examination.

\section{Ethical Statement:}

Permission was obtained from each patient before the study's enrollment. The study's protocol was supervised by an audit of the ophthalmology department, Faculty of Medicine, Al Azhar University.

\section{Study design:}

A prospective case series observational study was conducted on cases attending Ophthalmology Department of $\mathrm{Al}$ Azhar university hospital during the period from October 2018 to December 2019, who collected according to inclusion and exclusion criteria, examined and asked for permission, then photographed via OCT angiography device (TOPCON DRI OCT TRITON), this academic practice will evaluate macular perfusion in $6 \times 6 \mathrm{~mm}$ area. To guide early optimal treatment in subclinical diabetic retinopathy patients guarding against the risk of potential vision loss.

Patient's examination steps:

Patient's history was taken, Visual Acuity; Examined aided using (Snellen acuity chart), Intraocular pressure; measured using (Goldman applanation tonometry), Slit lamp examination; full eye examination; Anterior segment: Cornea, Anterior chamber, Iris, Pupil, and Lens, Posterior segment: Vitreous, and Fundus ex. (macula, vessels, optic nerve) by (Bio-microscopy Slit-lamp fundus examination using Volk 90 D lens). Then the patient asked for permission and photographed via OCTA device (Figure 1).

Methods: D-R-I OCT Triton Plus OCTA device (Topcon; Japan) (Figure 2).

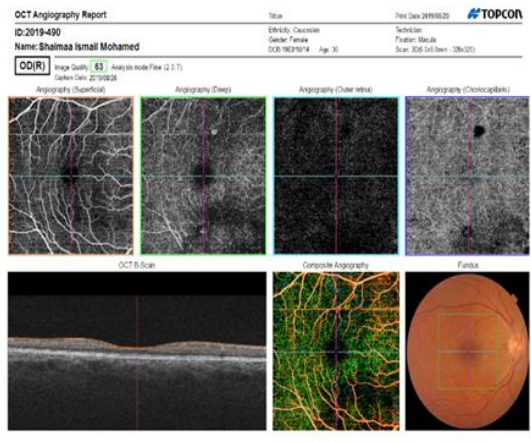

Fig.1: OCTA device.
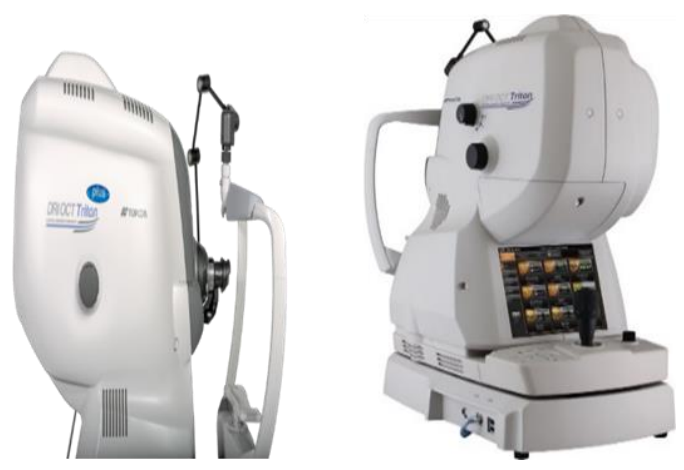

Fig. 2: D-R-I OCT Triton Plus OCTA device.

Inclusion criteria:

Controlled diabetes, Best Corrected Visual Acuity (BCVA): $6 / 18$ or better, Fundus picture (FP): normal or mild non-proliferative diabetic retinopathy (NPDR) with healthy macula, and patient's medical history: hypertensive or non-hypertensive, smoker or none.

\section{Exclusion criteria:}

Uncontrolled diabetes, BCVA: less than 6/18, FP: PDR or unhealthy macula (edema, exudates, drusen, hemorrhage), and patient's medical history: on Anticoagulants or Vasodilators or Vasoconstrictors medications.

\section{Statistical analysis:}

Abstracted data were compiled and analyzed using SPSS version 21 (SPSS Inc., Chicago, IL). Continuous variables are presented as means ( \pm standard deviation $[\mathrm{SD}]$ ), and categorical variables are presented using relative frequency distributions and percentages.

Continuous variables were compared using Student's t-test or the Mann-Whitney test, Categorical data were analyzed using the chi-square test, Yates' continuity correction, Fisher's exact test, and/or unadjusted odds ratios (ORs) as appropriate, and The p-value was considered significant as the following: Probability (P-value) with Statistical significance established at $\mathrm{p} \leq 0.05$. Fiures $3-18$ show the study findings.

RESULTS

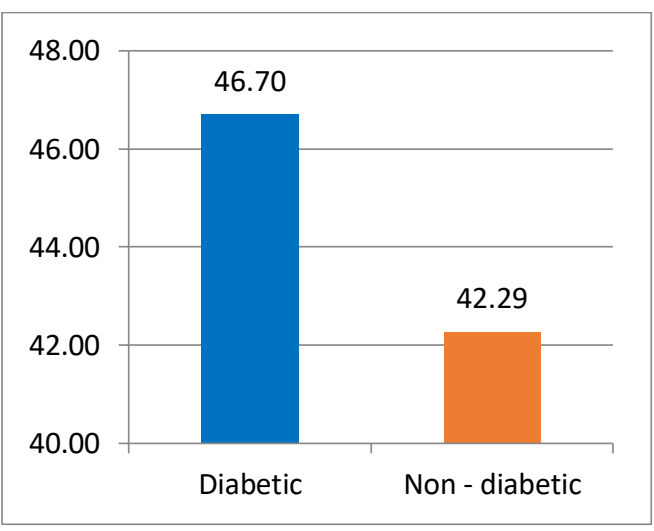

Fig. 3: Mean age of included patients. 


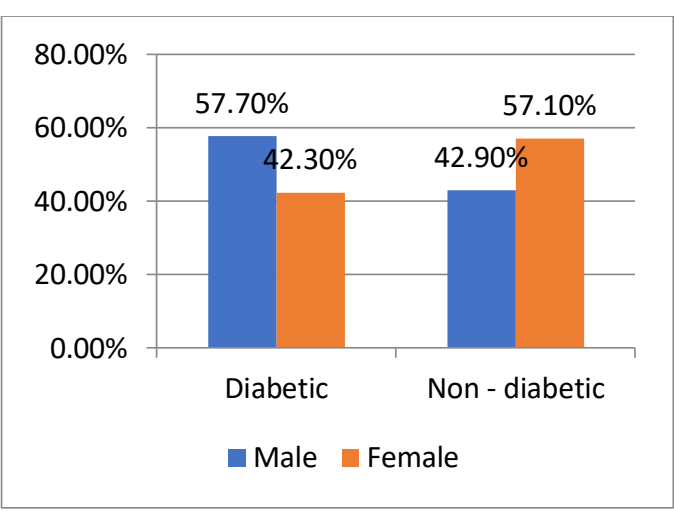

Fig. 4: Gender percentage in the included patients.

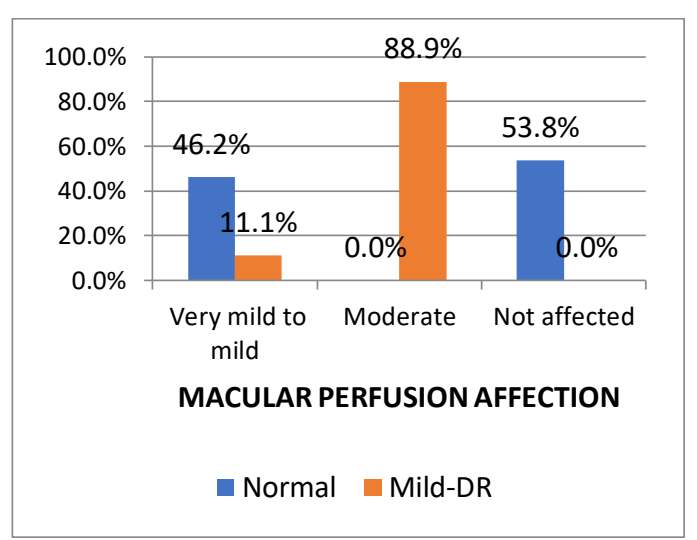

Fig. 5: Relation between Macular perfusion and Fundus picture.

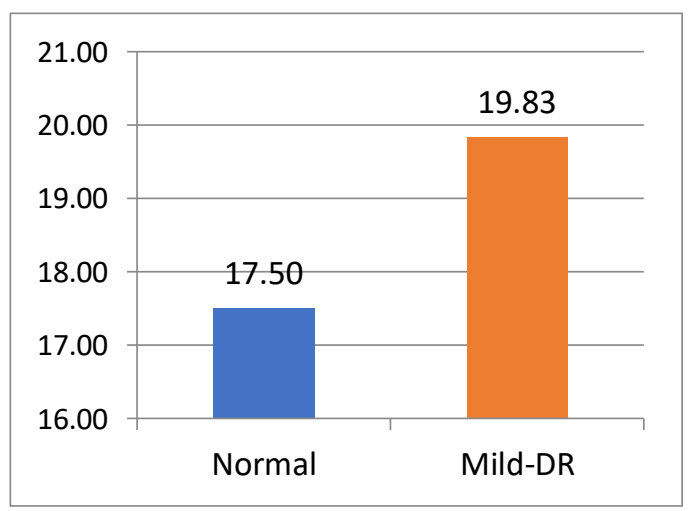

Fig. 6: Relation between mean Diabetes duration and Fundus picture.

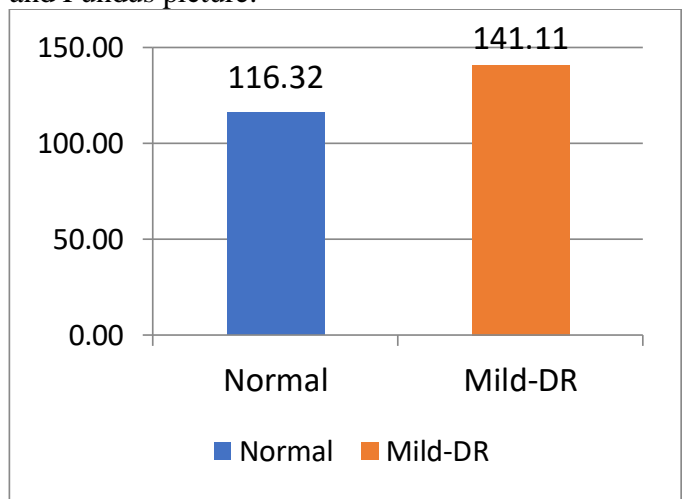

Fig. 7: Relation between mean Systolic Bp and Fundus picture.

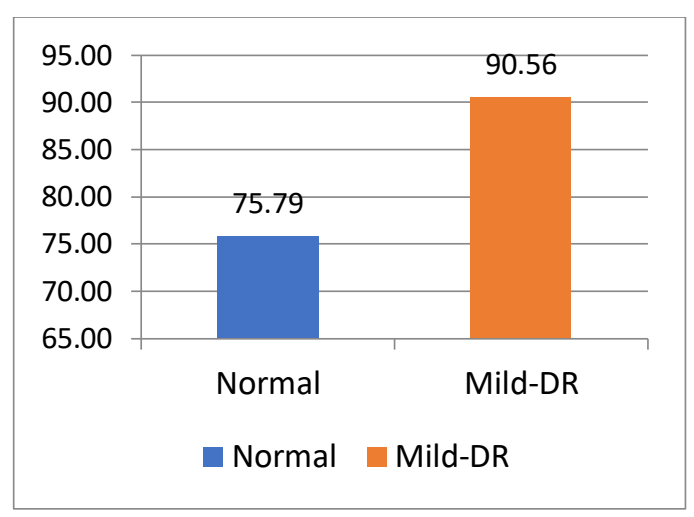

Fig. 8: Relation between mean Diastolic Bp and Fundus picture.

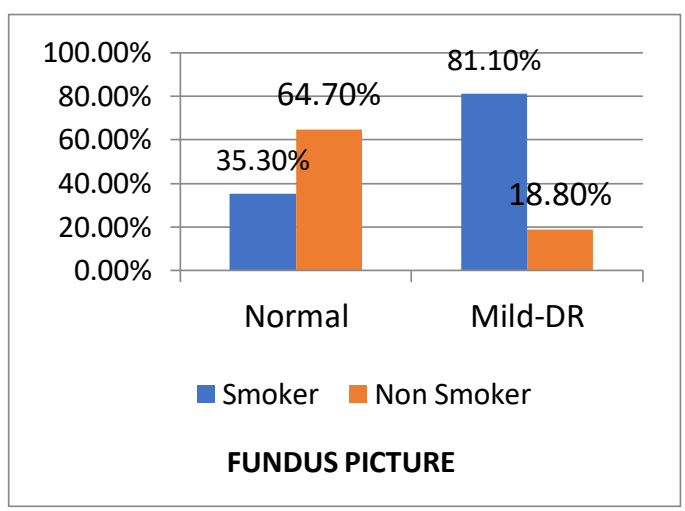

Fig. 9: Relation between smoking and the Fundus picture.

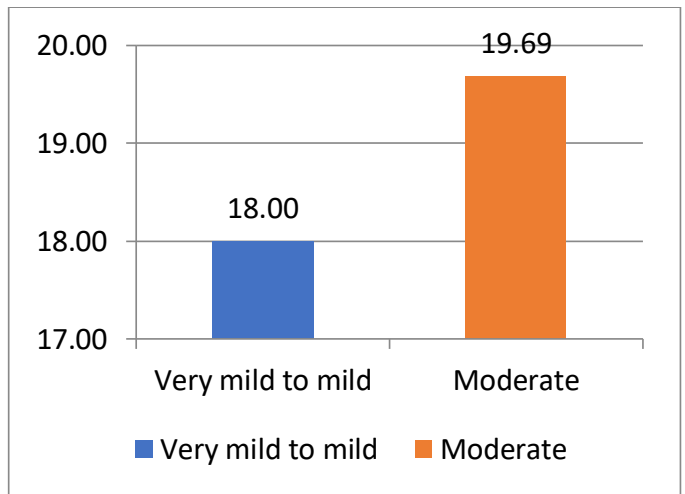

Figure 10: Relation between mean Diabetes duration and Macular perfusion.

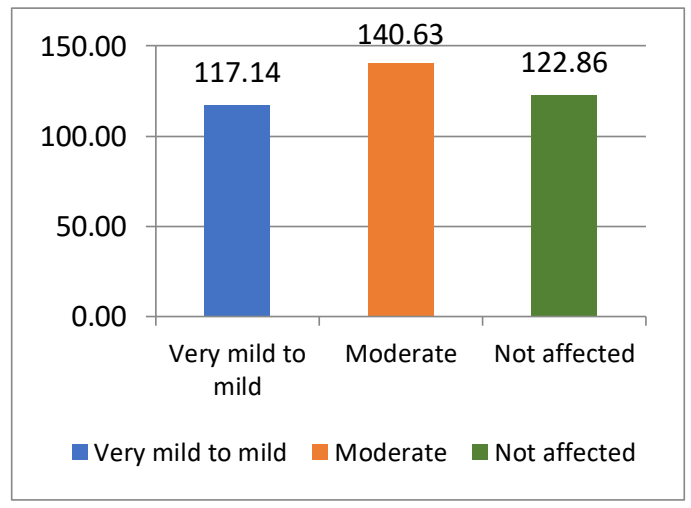

Fig. 11: Relation between mean Systolic Bp and Macular perfusion. 


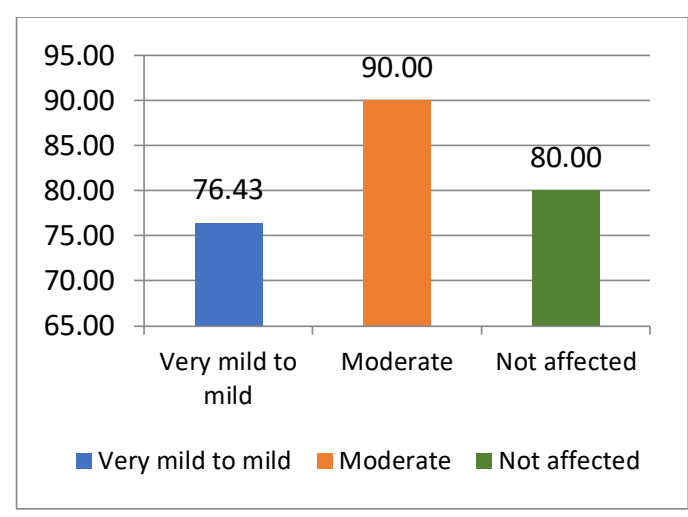

Fig. 12: Relation between mean Diastolic Bp and Macular perfusion.

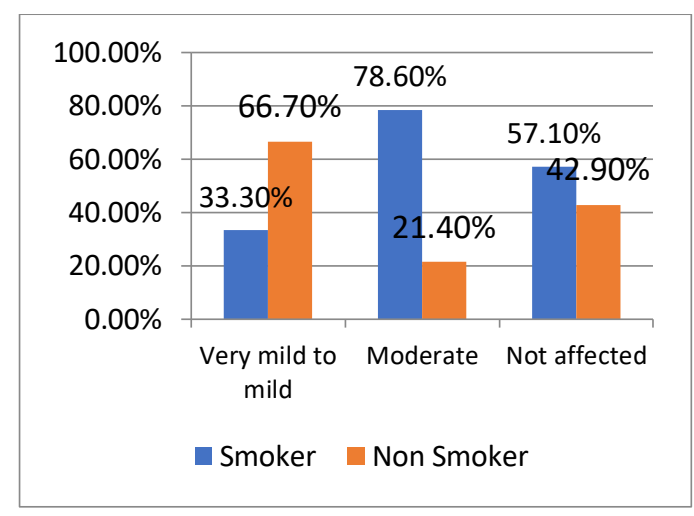

Fig. 13: Relation between the percentage of smoking and Macular perfusion.

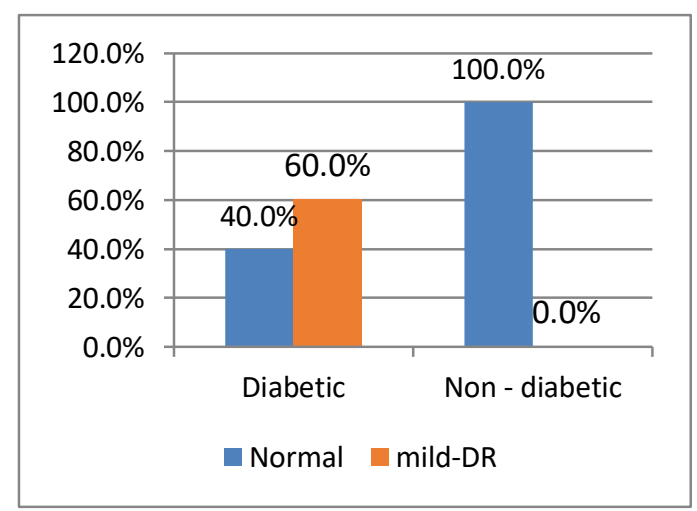

Fig. 14: Comparison between both groups according to the Fundus picture.

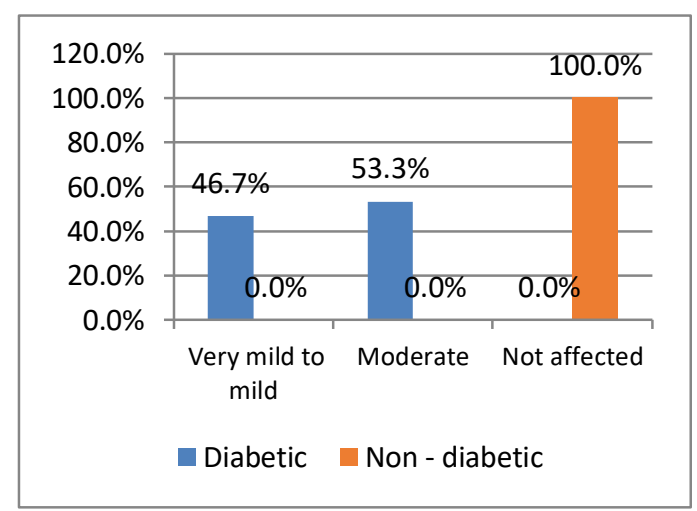

Fig. 15: Comparison between both groups according to Macular perfusion.

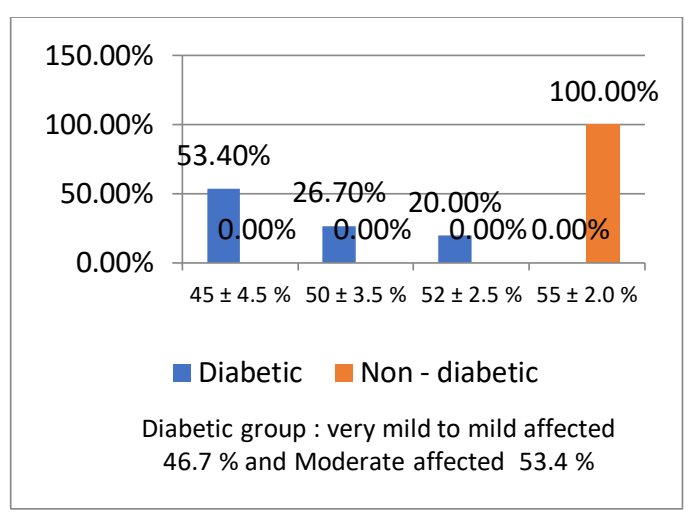

Fig. 16: Comparison between both groups according to Superficial Vascular Plexus - vessel density (SVPVD).

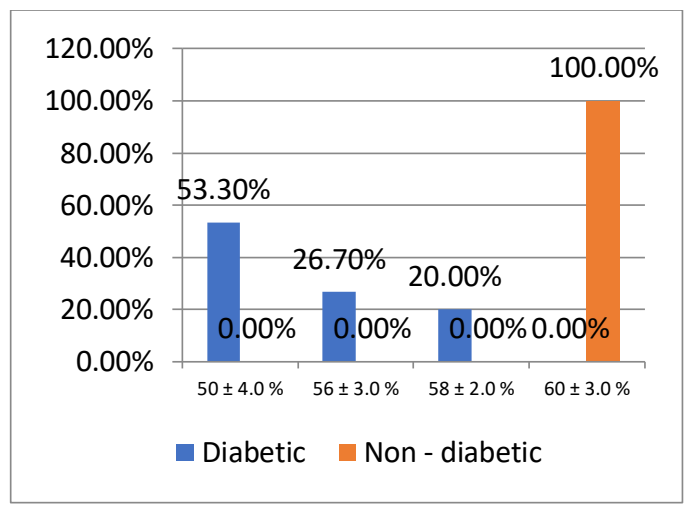

Fig. 17: Comparison between both groups according to Deep Vascular Plexus - vessel density (DVP-VD).

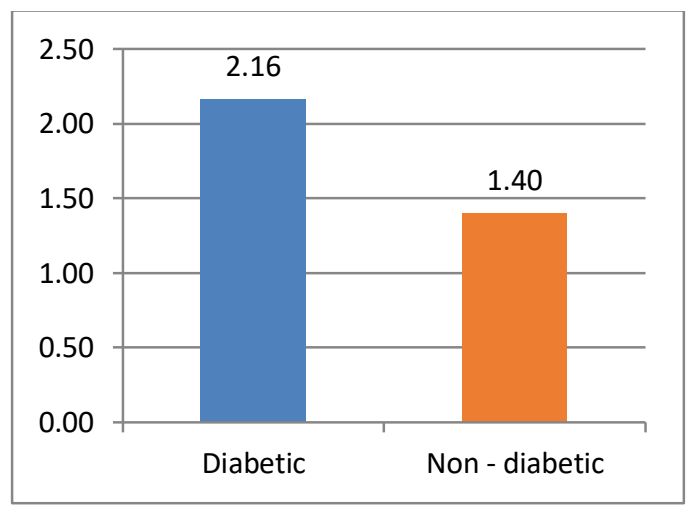

Fig. 18: Comparison between both groups according to FAZ Perimeter.

\section{DISCUSSION}

This is a prospective case series observational study that included 30 eyes of 26 diabetic patients and 14 eyes of 7 non-diabetic patients as a control group. In the diabetic group, the mean age was 46.7 (8.58), $57.7 \%$ of included patients were males, and $42.3 \%$ were females. In non - diabetic group, the mean age was 42.29 (5.12), $42.9 \%$ of included patients were males and $57.1 \%$ were females.

This is similar to Durbin et al. ${ }^{11}$ study which included 50 eyes from 26 patients with diabetes. In the distribution of sex among the diabetic group, 10 were women and 16 were men. 
estimated that patients with hypertension had more than double the risk of developing retinopathy after 10 years when compared with diabetic patients with normal blood pressure ${ }^{15}$.

In the present study, as regard diabetic group mean systolic blood pressure was measured among grades of macular perfusion affection which was $125(16.4)$ $\pm 10 \mathrm{mmHg}$ in very mildly affected to mildly affected patients and in moderately affected patients the mean was $140.6(11.8) \mathrm{mmHg}$ with a significant difference between groups $(\mathrm{P}$-value $=0.001)$. While mean diastolic blood pressure was in $81.6(11.6) \pm 10$ $\mathrm{mmHg}$ in very mildly affected to mildly affected patients, and in moderately affected patients the mean was 90(9.6) $\mathrm{mmHg}$ with a significant difference between groups $(\mathrm{P}$-value $=0.001)$. In agreement with Pascual-Prieto ${ }^{16}$ study in which there was a difference in macular perfusion between the hypertension groups. Analysis of macular perfusion showed significantly lower values in the mildly affected patients $(p=0.025)$. The control group had higher macular perfusion than the hypertensive groups.

\section{CONCLUSION}

Macular perfusion is affected by diabetes duration despite the clinical fundus picture. Early evaluation of perfusion is important in preventing prognosis of vision despite an uncomplaining patient, Hypertension control is important to factor in the prognosis of macular perfusion status, OCTA can be very beneficial in investigating diabetic patient who showed unexplained reduced best-corrected visual acuity, microvascular changes begin $1^{\text {st }}$ at the level of Superficial vascular plexus (SVP) then Deep vascular plexus (DVP), Microvascular changes in deep vascular plexus (DVP) and capillary drop out areas in DVP associated with worsening BCVA significantly more than changes in superficial vascular plexus (SVP), and Gender is not a significant risk factor.

\section{REFERENCES}

1. Korobelnik JF, Do DV, Schmidt-Erfurth U, et al. Intravitreal aflibercept for diabetic macular edema. Ophthalmology, 2014; 121(11):224754.

2. Miyamoto K, Khosrof S, Bursell SE, et al. Prevention of leukocytosis and vascular leakage in streptozotocin-induced diabetic retinopathy via intercellular molecule-1 inhibition. Proc Natl Acad Sci U S A., 1999; 96: 10836-41.

3. Ip MS, Domalpally A, Sun JK, et al. Long-term effects of therapy with ranibizumab on diabetic retinopathy severity and baseline risk factors for worsening retinopathy. Ophthalmology, 2015; 122: 367-74.

4. Chung EJ, Roh MI, Kwon OW, et al. Effects of macular ischemia on the outcome of intravitreal bevacizumab therapy for diabetic macular edema. Retina, 2008; 28: 957-63.

5. Nakagawa $\mathrm{S}$, Oishi A, Ogino $\mathrm{K}$, et al. Association of retinal vessel attenuation with visual function in eyes with retinitis pigmentosa. Clinical Ophthalmology, 2014; 8: 1487-93.

6. Hwang TS, Jia Y, Gao SS, et al. Optical coherence tomography angiography features of diabetic retinopathy. Retina, 2015; 35: 2371-6.

7. Di G, Weihong $\mathrm{Y}$, Xiao Z, et al. A morphological study of the foveal avascular zone in patients with diabetes mellitus using optical coherence tomography angiography. Graefes Arch Clin Exp Ophthalmology, 2016; 254: 873-9.

8. Bhanushali D, Anegondi N, Gadde SG, et al. Linking retinal microvasculature features with severity of diabetic retinopathy using optical coherence tomography angiography. Invest Ophthalmology Vis Sci., 2016; 57: 519-25.

9. de Carlo TE, Romano A, Waheed NK, et al. A review of optical coherence tomography angiography (OCTA), International Journal of Retina and Vitreous 2015 1:5.

DOI 10.1186/s40942-015-0005-8

10. Choi W, Mohler KJ, Potsaid B, et al. Choriocapillaris and choroidal microvasculature imaging with ultrahigh speed OCT angiography. PLoS ONE., 2013; 8(12):81499.

11. Durbin MK, An L, Shemonski ND, et al. Quantification of retinal microvascular density in optical coherence tomographic angiography images in diabetic retinopathy. JAMA Ophthalmology, 2017; 135(4): 370-6.

12. Varma R, Macias GL, Torres M, et al. Biologic risk factors associated with diabetic retinopathy: the Los Angeles Latino Eye Study. Ophthalmology, 2007; 114:1332-40.

13. Kawasaki R, Laporte R, Tojo K, et al. "Factors associated with non-proliferative diabetic retinopathy in patients with type 1 and type 2 diabetes: the Japan Diabetes Complication and its Prevention prospective study (JDCP study 4). Diabetology International, 2019; 10(1): 311 .

14. Zhang $\mathrm{X}$, Saaddine JB, Chou CF, et al. Prevalence of diabetic retinopathy in the United States, 2005-2008. JAMA., 2010; 304:649-56

15. van Leiden HA, Dekker JM, Moll AC, et al. Risk factors for incident retinopathy in a diabetic and nondiabetic population: the Hoorn study. Arch Ophthalmology, 2003; 121:245-51.

16. Pascual-Prieto J, Burgos-Blasco B, Ávila Sánchez-Torija M, et al. Utility of optical coherence tomography angiography in detecting vascular retinal damage caused by arterial hypertension. Eur J Ophthalmology. 2019.. doi: 10.1177/1120672119831159. 